\title{
Unusual Posttraumatic Vascular and Cerebral Injuries in Young Patients
}

\author{
Emilia Marciuc ${ }^{1,2}$, M. Barcan ${ }^{1}$, B. Dobrovăț ţ, ${ }^{1,2}$ Roxana Popescu ${ }^{1,2}$, \\ Cornelia Tudorache ${ }^{2}$, Danisia Haba ${ }^{1,2}$ \\ ${ }^{1}$ Department of Radiology and Medical Imaging of Emergency Hospital „Prof. Dr. N. Oblu”, \\ Iasi, ROMANIA \\ 2"Grigore T. Popa" University of Medicine and Pharmacy, Iasi, ROMANIA
}

\begin{abstract}
Blunt carotid artery injury is a relatively rare but potentially lethal injury that predominantly occurs in high-impact mechanisms such as motor-vehicle collision. Any simptoms or neurological deficits following a multiple trauma patient mandates a thorough evaluation and consideration of BCI. This is a case report on two young patients with neurologic simptoms developed after blunt trauma in the cervical region. Both patients had left internal carotid artery dissection diagnosed on a CTangiography, followed by middle cerebral artery territory infarction. Although it can be a delayed onset with no signs of vascular demage, we believe that, by implementing a protocol with additional imaging for early detection, we can prevent a devastating outcome.
\end{abstract}

Key words: cerebral infarction, internal carotid artery dissection, trauma

\section{Introduction}

Blunt carotid artery injury is a rare condition first described by Yamada et al. in 1967(Yamada S 1967)that predominantly occurs in high-impact mechanisms such as motor-vehicle accidents. It is observed in $1 \%$ $2.6 \%$ of blunt trauma cases and it's associated with high rates of ischemic stroke (up to $60 \%$ ) and mortality (19-43\%)(Thomas S. Lee 2014). Because it's a life-threatening condition, early detection is mandatory and requires a good understanding of injury mechanism and thorough evaluation which can be a key point in offering the patient a favorable outcome.

\section{Case report}

\section{Case 1}

A 27 years old female patient, motorvehicle collision victim (driver) was brought to the emergency room presenting headache, dizziness and multiple contusions including a cervical region blunt trauma. An initial non contrast head CT was performed that showed no posttraumatic lesions. After 24 hours, the 
patient's condition worsened and a head MRI revealed a T2WI, FLAIR, T2* GE high-signal and T1WI low-signal in the cortical and deep white matter cerebral area of the left frontal, temporal and parietal lobes. The axial diffusion-weighted image demonstrates high signal in the same area with corresponding low signal on the apparent diffusion coefficient (ADC) maps, consistentwith true restricted diffusion and an acute/subacute infarction in the middle cerebral artery territory (Figure 1).

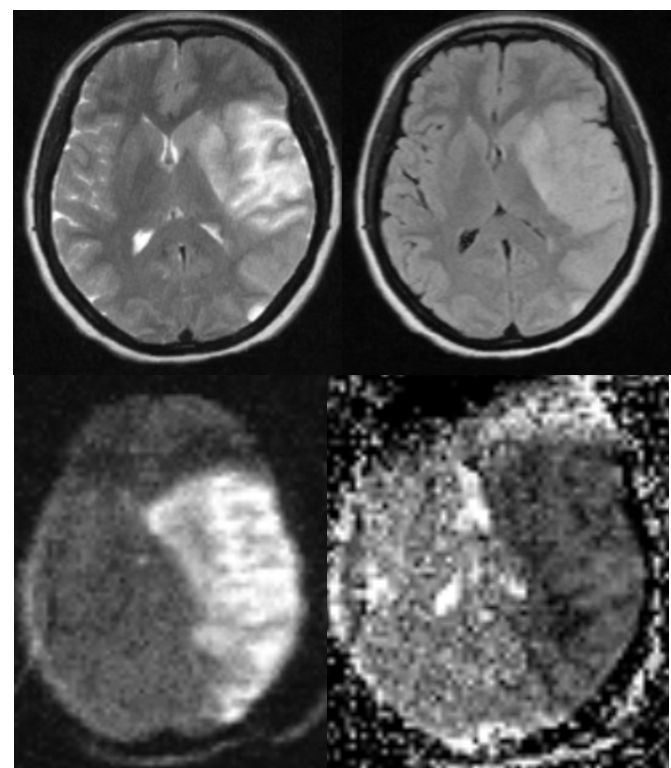

Figure 1 - Acute/subacute infarction in the middle cerebral artery territory (a. Axial T2WI, b. Axial FLAIR, c. DWI, d. ADC Map)

Another area with same aspects, with a diameter of $13 \mathrm{~mm}$ in the right cerebellar hemisphere is shown, consistent with a recent lacunar infarction or a diffuse axonal lesion (Figure 2).

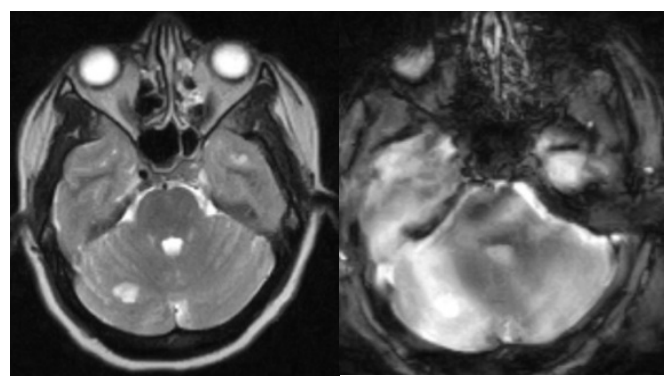

Figure 2 - Recent lacunar infarction or a diffuse axonal lesion (a. Axial T2WI, b.Axial T2*)

Also, in the SE sequences, no signal-void in the cervical, petrous, lacerum, cavernous and supraclinoidiansegments of left internal carotid artery is visualized, aspect which indicates for a slow flow or occlusion of left ACI (Figure 3 a,b). Same absence of signalvoid for V3 and V4 segments of right vertebral artery (Figure $3 \mathrm{c}$ ).

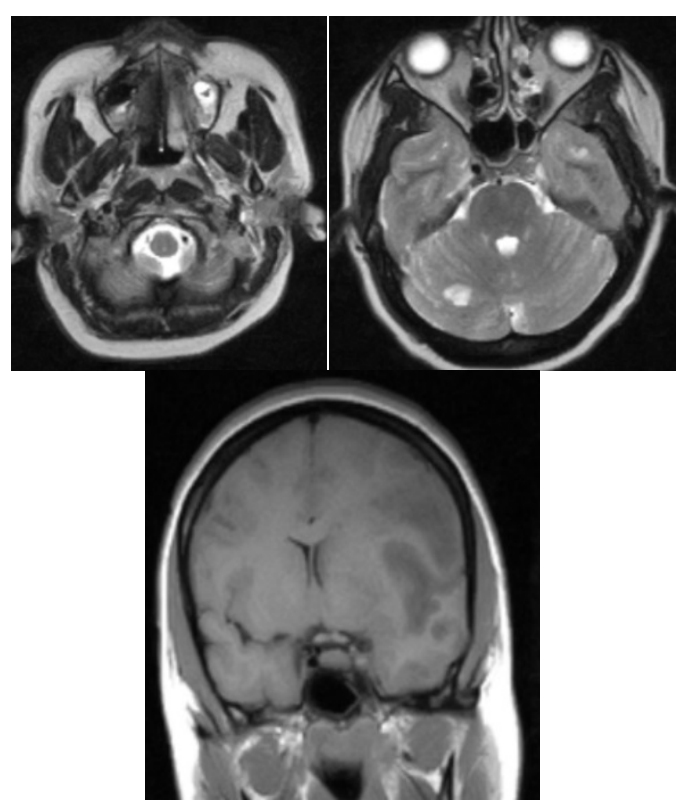

Figure 3 - Spin Echo sequences (a,b. Axial T2WI, c. Coronal T1WI) 
A head and neck CT Angiography was recommended that showed complete occlusion of left ACI right above bifurcation and partially occlusion of right vertebral artery in V3 and V4 segments (Figure 4).

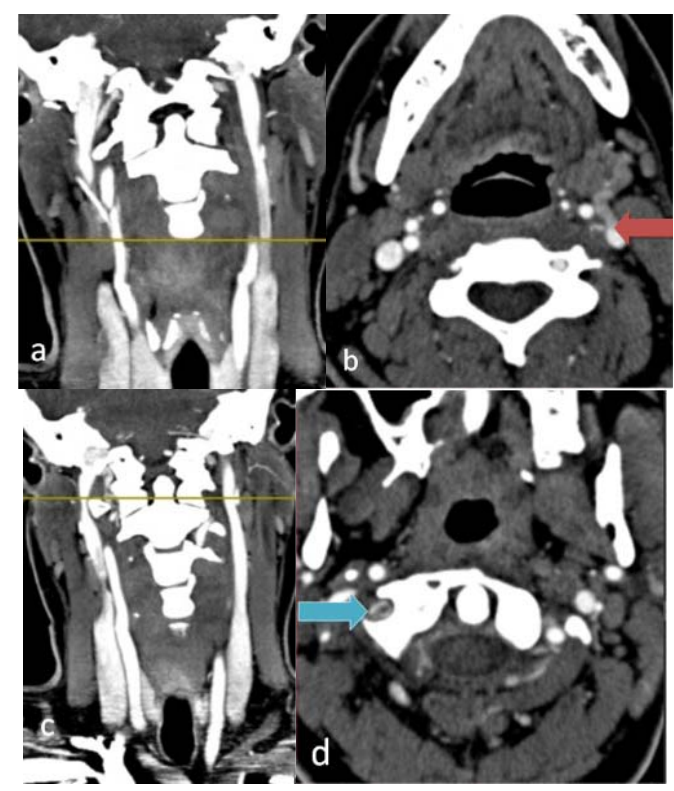

Figure 4 - Complete occlusion of leftACI right above bifurcation $(a, b)$ and partially occlusion of right vertebral artery in V3 and V4segments (c,d).

On non-contrast head CT scan was observed a midline shift of $9 \mathrm{~mm}$ which was an indication for a decompressive craniectomy (Figure $5 \mathrm{a}, \mathrm{b}$ ). The following CT scans showed the ischemic area throughout all stages. After 4 month the patient came back for follow-up (Figure $5 \mathrm{c}$ ) and presented with improvements in motor control and regained the capacity to carry out activities of daily living.

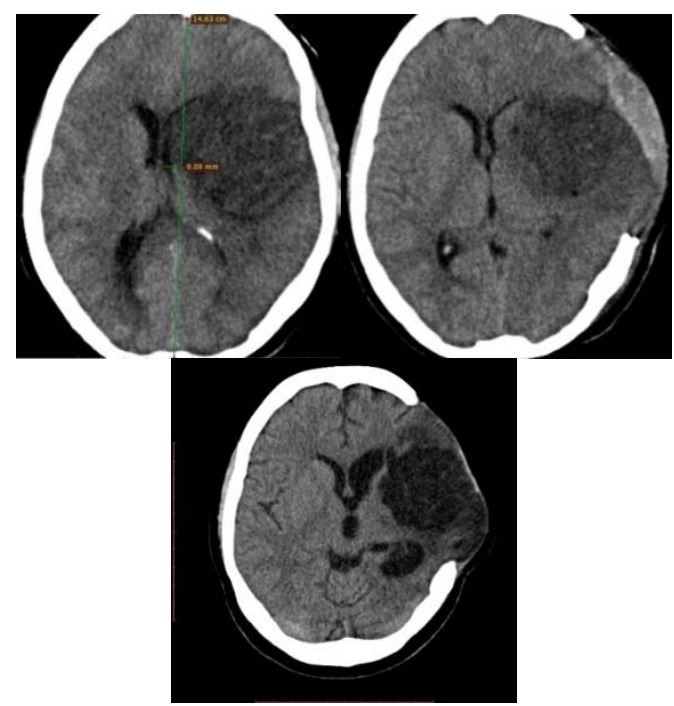

Figure 5 - Pre-op (a), post-op (b) and after 4 months(c) radiological aspects

Case 2

A 17-year old male patient suffered a cervical trauma after getting his neck caught between the doors of an elevator with no immediate symptoms. After approximatively 2 hours, the patient presented neurologic symptoms (aphasia, motor impairment) and a left cervical hematoma. After another 3 hours is brought to the emergency room where a non-contrast head CT scan is performed, which reveals a loss of grey-white matter differentiation,hypoattenuation of deep nuclei, cortical hypodensity with associated parenchymal swelling and resultant gyral effacement in the left frontal, temporal and parietal lobes. Also the hyperdense middle cerebral artery sign (Figure 6 a)andmiddle cerebral artery dot sign are present (Figure 6 b), all aspects are consistent with an acute stroke in the left middle cerebral artery territory. Carotid ultrasound was performed and detected a free-floating thrombus of the 
left carotid artery (Figure $7 \mathrm{a}, \mathrm{b}$ ). The vascular neurosurgical team was consulted and an endovascular treatment with stenting of the carotid artery was immediatly performed (Figure 8), but the evolution of the patient was variable. The post-surgery head CT scan showed a massive edema with a midline shift of $10 \mathrm{~mm}$ (Figure 9a), so a decompressive craniectomy (Figure 9b) was indicated, but the outcome of the patient was unfortunate with exitus in the next 72 hours.

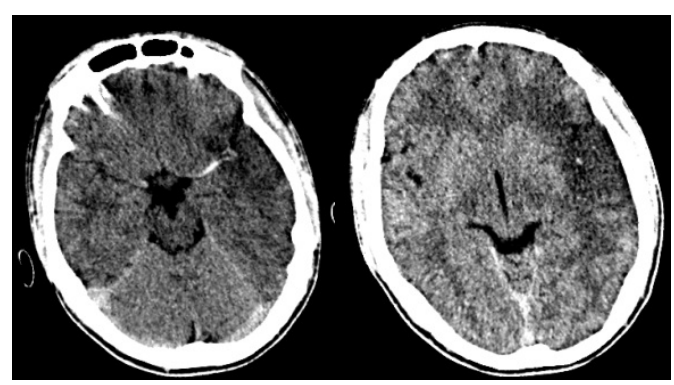

Figure 6 - Acute stroke in left middle cerebral artery territory - hyperdense middle cerebral artery sign (a) and middle cerebral artery dot sign (b).

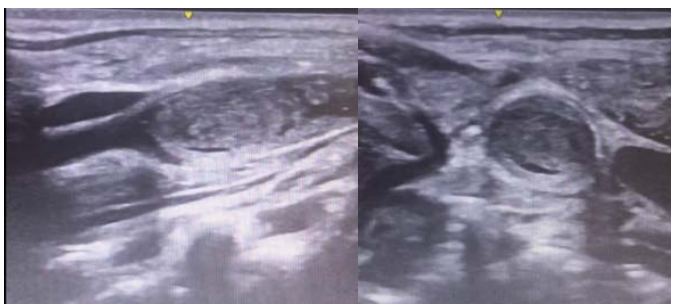

Figure 7 - US free-floating thrombus of the left carotid artery (a. Sagital view, b. Transversal view)

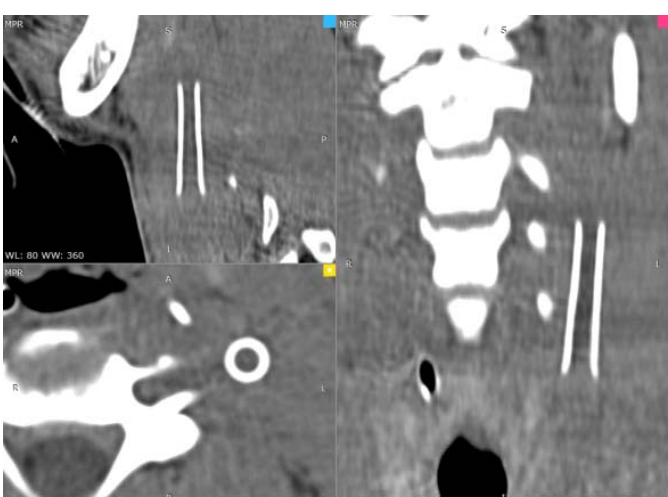

Figure 8 - Endovascular treatment with stenting of the left carotid artery

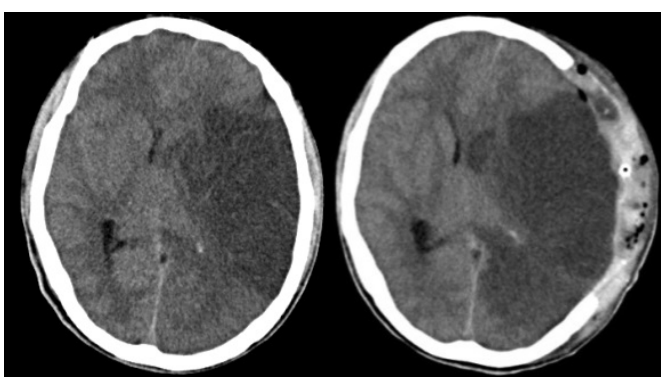

Figure 9 - Pre-op (a) and post-op (b) radiological aspects.

\section{Discussions}

Blunt carotid artery injuries are uncommon and considered life-threatening because of the delayed onset of neurologic deficits which can lead to a poor outcome for patients. Therefore, knowledge regarding the injury mechanism may be of aid in making an earlier diagnosis and treatment. Mechanisms of injury include a direct blow to anterolateral aspect of the cervical region, hyperextension, flexion or rotation of the neck, even the vasocompression of the internal carotid artery between the cervical spine and mandible (George Galyfos 2016). These forces are present in the setting of motor-vehicle collisions, cervical spine or 
mandible fractures, chiropractic manipulation (Thomas S. Lee 2014) and can lead to small lesions of the vessel wall which may result in intimal tears, creating the potential for vessel dissection (separation of vesselwalltissuelayers) or intramural thrombus formation, leading to vessels stenosis or complete occlusion (Thomas S. Lee 2014). These events lead to failure of brain perfusion and ischemic stroke which can have different outcomes even in young patients with no other comorbidities.

These patients were both diagnosed with carotid artery dissection but the timing of diagnosis was different. In the first case, a high index of suspicion given by the traumatic mark in the cervical region and the mechanism of injury should be an important determinant in ordering a CT angiography despite the poor clinical signs. Most likely, the internal carotid artery dissection was caused by the hyperextension of the neck in the moment of impact combined with the seatbelt injury in the lateral aspect of the neck. Although the patient wasn't a good candidate for endovascular treatment, the outcome was satisfying considering the reduction in the extent of neurologic impairment.

Another particularity of the case was the dissection of right vertebral artery induced also by shearing forces in hyperextension/flexion during collision but with no consequences for the posterior cerebral artery territory of the brain. Traumatic blunt vertebral artery injury is a rare condition, the reported incidence is variable in the literature $(0.5-2 \%$ of all trauma patients)(Fassett DR 2008). Motor vehicle accidents are the first cause of this type of injury but the V2 segment is most commonly affected while in V3 and V4 segments the artery has a greater mobility and is more resistant to blunt trauma(R. M. deSouza 2011). Most commonly, these dissections are sub-intimal and cause stenosis (48\%) or occlusion (35\%) (Karthikeyan Y.R. 2017).

In the second case, there was an earlier onset of neurologic deficits which established the diagnosis of carotid artery dissection and an endovascular stenting was possible within a few hours after presenting to the emergency room. However, the outcome was unfortunate.

Carotid artery dissection is a rare but lifethreatening condition that should be considered in patients with a mechanism of injury that places these major vessels at risk. Knowledge regarding the mechanism of injury may facilate an early diagnosis with major impact in the patients' prognosis. A study conducted by Miller PR has identified specific findings that may suggest a blunt carotid artery injury including C-spine fracture, Le Fort II/III facial fracture, Horner's syndrome, skull base fracture involving the foramen lacerum and soft tissue injury in the cervical region. (Miller PR 2002). Although, digital substraction angiography (DSA) is the gold standard for the diagnose of a possible dissection(Thomas S. Lee 2014), computed tomography angiography (CTA) is more accessible, can be easily performed in cases of severe trauma and can be a useful diagnosis tool. 


\section{Conclusions}

Patients with traumatic blunt carotid artery dissection are a subgroup of cases which demand special management in an interdisciplinary setting and additional imaging in order to provide a good outcome, especially in young patients. Despite the subtle clinical picture at the beginning, the morbidity and mortality rates are high, so the early detection is a key factor for an optimal result.

\section{Correspondence}

Dr. Bogdan Dobrovăț, University of Medicine and Pharmacy "Gr. T.Popa" Iasi

Email:bogdan.dobrovat@yahoo.com

\section{References}

1. Fassett DR, Dailey AT, Vaccaro AR. "Vertebral artery injuries associated with cervical spine injuries: a review of the literature." J Spinal Disord Tech, 21(4) : 252-258, 2008.

2. George Galyfos, Konstantinos Filis, Fragiska Sigala, Argiri Sianou. "Traumatic Carotid Artery Dissection: A
Different Entity without Specific Guidelines." Vascular Specialist International, 32(1): 1-5, 2016.

3. Karthikeyan Y.R., Sanjeev Chopra, Somnath Sharma, Devendra Purohit. "Post traumatic vertebro basilar dissection: case report and review of literature." Romanian Neurosurgery, 31(3): 310-315, 2017.

4. Lee TS, Ducic Y, Gordin E, Stroman D. "Management of carotid artery trauma." Craniomaxillofac Trauma Reconstruction: 175-189, 2014.

5. Miller PR, Fabian TC, Croce MA, Cagiannos C, Williams JS,Vang M, Qaisi WG, Felker RE, Timmons SD. "Prospective screening for blunt cerebrovascular injuries. Analysis of diagnostic modalities and outcomes." Ann Surg, 236(3): 386-395, 2002.

6. R. M. deSouza, M. J. Crocker, N. Haliasos, A. Rennie, A. Saxena. "Blunt traumatic vertebral artery injury: a clinical review." Eur Spine J, 20(9): 1405-1416, 2011.

7. Thomas S. Lee, Yadranko Ducic, Eli Gordin, David Stroman. "Management of Carotid Artery Trauma." Craniomaxillofac Trauma Reconstruction, 7(3): 175189, 2014.

8. Yamada S, Kindt GW, Youmans JR. "Carotid artery occlusion due to nonpenetrating injury." J Trauma, 192(1): 333-342, 1967. 